\title{
Effect of Bile Salts on Self-Assembly and Construction of Micro-/nanomaterials
}

\author{
JIAO Jianmei ${ }^{1}$, XU Guiying ${ }^{2}$, XIN Xia ${ }^{1,2,{ }^{*}}$ \\ ${ }^{1}$ National Engineering Technology Research Center for Colloidal Materials, Shandong University, Jinan 250100, P. R. China. \\ ${ }^{2}$ Key Laboratory of Colloid and Interface Chemistry, Ministry of Education, Shandong University, Jinan 250100, P. R. China.
}

\begin{abstract}
Bile acid salts, which are regarded as anionic steroid biosurfactants, have been widely used in the preparation of novel nanomaterials owing to their special amphiphilic skeleton structure, unique physical and chemical properties, good biocompatibility, and environmental friendliness. They can participate in the supramolecular self-assembly to form ordered aggregates in solution, and can be used as a template for the preparation of micro and nanomaterials. In this manuscript, we present an overview of our research work based on the effect of bile salts on the self-assembly of micro and nanomaterials along with related research done worldwide. The first section introduces the effect of small biological molecules such as amino acids, on the aggregation behavior of bile salts, wherein the interaction between amino acids and cholate has been studied extensively. Many in vivo and in vitro studies have been carried out mainly focusing on solutions and interfaces. Second part of this manuscript summarizes the research progress on the construction of supramolecular gels based on bile salts, including amino acids, rare earth salts, Graphene Oxide (GO), and surfactants. Cholic acid sodium, sodium deoxycholic acid, and lithocholic acid sodium cholic acid salt have special steroidal parent skeleton, which is much more complicated than the alkane surfactant, enabling them to self-assemble to form gel via non-covalent interactions such as van der Waals force, hydrogen bonding, and hydrophobic effect. Also addition of small molecules and other organic/inorganic fillers can increase the mechanical strength of the gels. Last part describes the formation of micro and nanomaterials by self-assembly in presence of bile salts, especially about their interaction with dye molecules, wherein the formed complex usually has novel and ordered microstructures. The interaction between surfactants and dye molecules are mainly driven by electrostatic forces, hydrogen bonding, and van der Waals force. Dye molecules are considered to be an ideal substrate for constructing functional nanomaterials and as biosurfactant, bile salts are often used to assist in the synthesis of micro and nanomaterials. In order to get a more comprehensive and in-depth understanding of the preparation of micro and nanomaterials in presence of bile salts, this article provides a solid foundation to explore future applications. Although the participation of bile salts in supramolecular self-assembly and in the preparation of various functional nanomaterials has not been studied much, the current research focuses on the template function of bile salt and ionic self-assembly. However, the biological and physiological aspects of bile salts are low; therefore the function of bile salts still needs to be probed.
\end{abstract}

Key Words: Bile salt; Self-assemly; Aggregation behavior; Gels; Micro and nano materials 


\title{
胆酸盐参与的自组装及微纳米材料制备
}

\author{
焦建梅 ${ }^{1}$, 徐桂英 ${ }^{2}$, 辛霞 $1,2,{ }^{*}$ \\ 1 山东大学, 国家胶体材料工程技术研究中心, 济南 250100 \\ 2 山东大学, 胶体与界面化学教育部重点实验室, 济南 250100
}

\begin{abstract}
摘要: 胆酸盐类物质可看作是一类阴离子型甾族生物表面活性剂, 鉴于其特殊的两亲性骨架结构、独特的物理化学性质 及其良好的生物相容性和环境友好性, 其在溶液中能够参与超分子自组装形成有序聚集结构, 且可以作为模板在微纳材 料制备领域有着重要应用。本文结合我们课题组的研究工作, 综述了近期国内外相关研究, 详细介绍了生物小分子氨基 酸对胆酸盐聚集行为的影响、胆酸盐参与形成的超分子凝胶及胆酸盐参与构筑的微纳米材料制备等方面的研究进展, 以 期对胆酸盐参与的自组装及微纳米材料制备领域的研究有更全面更深入的了解，为后续的应用研究提供坚实的基础。
\end{abstract}

关键词: 胆酸盐; 自组装; 聚集行为; 凝胶; 微纳米材料 中图分类号：0647

\section{1 引言}

胆酸盐(bile salts)是生物体内广泛存在的一类 活性物质, 在机体中具有非常重要的生理功能。 主要包括调节胆固醇代谢、增强肤脂肪酶的活性、 防止胆石生成, 同时促使脂肪乳化成脂肪微粒、 促进脂肪酸和脂溶性维生素(A、D、E、K)的吸收 等1。传统的表面活性剂是线性结构, 包括亲水和 疏水两种基团, 亲水基团一般由带电的离子基团 或不带电的极性基团构成, 疏水基团由长链烃基 构成。由于胆酸盐类物质也同时具有疏水和亲水 两种基团, 因此其也可以看作是一类阴离子型甾 族生物表面活性剂(biosurfactant), 而且与传统表 面活性剂相比, 胆酸盐类生物表面活性剂具有以 下特征：(1)面形结构(图1), 具有极性面和非极性 面。极性面是亲水面, 由羟基、羧基或羧基与甘 氨酸 (glycine)、牛磺酸 (taurine) 形成的轭合物 (conjugates)构成, 位于 $\alpha$ 面(凹面); 非极性面是疏 水面, 含有一个刚性的、接近平面的胆甾类疏水 性骨架, 主要由两个角甲基及甾族环构成, 分布 在 $\beta$ 面(凸面); 整个分子偏转, 形成近似弓形的结 构, 使胆酸盐具有两亲分子的性质。(2)所占空间 体积较大, 刚性较强。(3)既具有生物表面活性剂 的性质, 无毒、可降解、生物兼容性好, 又具有

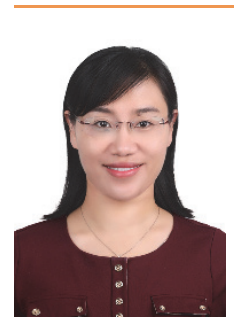

辛霞, 女, 1981 年生。山东大学国 家胶体材料工程技术研究中心, 副 教授, 硕士生导师。研究领域: 胶 体与界面化学, 包括表面活性剂与 小分子、聚合物之间的相互作用; 荧光材料的合成及其在两亲分子 组装体中的性能调控。
阴离子表面活性剂的性质2,3。因此, 鉴于胆酸盐 特殊的两亲性空间骨架结构、独特的物理化学性 质及其良好的生物相容性和环境友好性, 使其在 超分子自组装、碳纳米管分散 ${ }^{4} 、$ 分子识别 ${ }^{5}$ 药物 缓释、制备生物材料和环保材料等领域都有非常 重要的应用。

自组装在自然界中广泛存在。自组装法是近 年来兴起的一种简便而高效的制备方法, 是指构 筑基元(如分子、离子等)在一定条件下通过静电引 力、氢键、配位键、 $\pi-\pi$ 堆叠作用和分子识别等相 互作用形成多种有序聚集体的过程, 在功能性结 构的构筑和阐明复杂的生命演化过程方面起着至 关重要的作用 6,7 。胆酸盐具有超分子自组装能力, 可以自组装成囊泡、纳米管、纳米纤维、纳米螺 旋等特殊的纳米结构 $8-10$ 。本文基于我们课题组的 研究成果, 结合国内外相关研究, 详细介绍了氨 基酸对胆酸盐聚集行为的影响、胆酸盐参与形成 的超分子凝胶及胆酸盐参与构筑的微纳米材料的 制备等方面的研究进展, 以期对该领域的研究有 更全面更深入的了解, 为后续的应用研究提供坚 实的基础。

\section{2 氨基酸对胆酸盐聚集行为的影响}

胆酸盐与蛋白质在生物体内广泛存在, 两者 之间不可避免地发生相互作用 ${ }^{11-15}$ 。其中, 氨基酸 是蛋白质的基本组成单元, 可以反映球状蛋白的 一些结构特征, 因其分子量较小、结构较简单的 特点通常被用来作为研究结构较为复杂的蛋白 质的模型。近年来, 氨基酸和胆酸盐的相互作用 是一类研究比较多的话题, 很多体内和体外的研 究都围绕此课题展开, 研究主要集中于溶液与界 


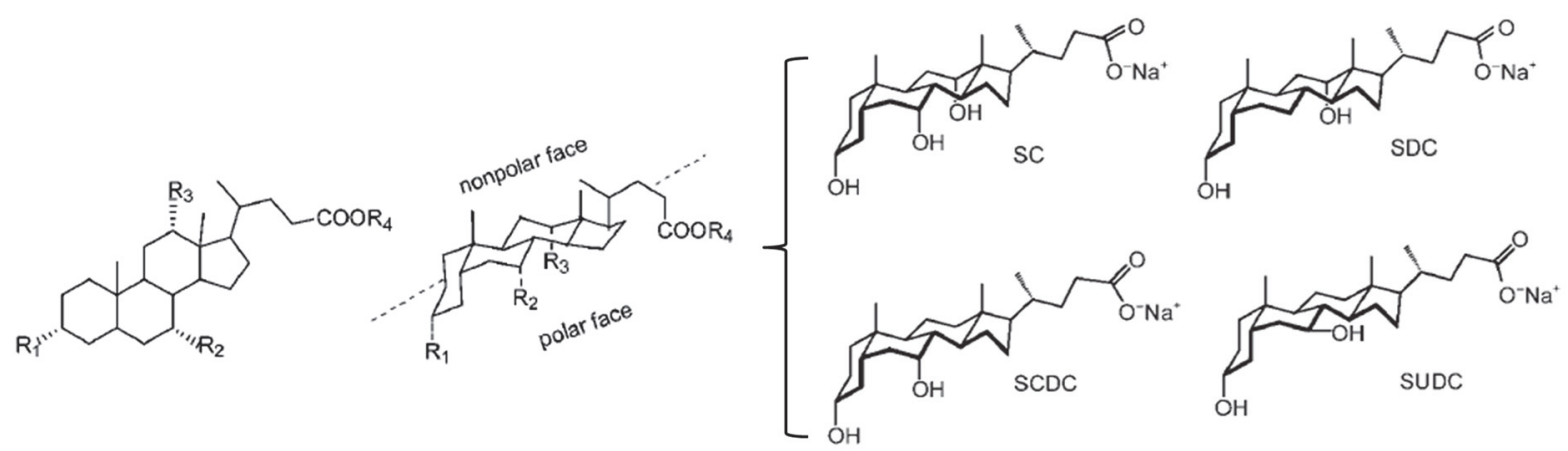

图 1 胆酸盐的结构通式及常见的胆酸盐结构 ${ }^{2}$

Fig. 1 The general structures of bile salts and structures of common bile salts ${ }^{2}$.

面中。

Smal ${ }^{16}$ 和Carey ${ }^{17}$ 等认为胆酸盐在水溶液中的 聚集过程是一个逐级缔合过程, 并提出了初级-二 级聚集模型。胆酸盐分子首先通过疏水作用形成 聚集数小于 10 的初级胶束, 而后这些初级胶束通 过着基间的氢键作用发生聚集, 形成更大的二级 胶束。Kawamura等 ${ }^{18}$ 进一步将该模型进行了扩展, 提出胆酸盐分子是以背对背的方式排布的。而 Giglio等 19,20 提出了螺旋模型, 认为胆酸盐分子间 主要通过极性作用连接起来, 疏水基团朝向水相, 而亲水面朝向聚集体内部。

我们课题组通过测定萠的稳态苂光光谱, 研 究了三种碱性氨基酸L-赖氨酸(L-Lys)、L-精氨酸 (L-Arg) 和 L- 组氨酸 (L-His) 对脱氧胆酸钠 (sodium deoxycholatCe, $\mathrm{NaDC}$ )在溶液中聚集行为的影响 ${ }^{21}$ 。 结果表明, 氨基酸的加入可以降低 $\mathrm{NaDC}$ 在水溶液 中的微极性和 $\mathrm{cac}$ 值, 而体系的聚集数也显著增 加。L-Lys对微极性、 cac和聚集数的影响最大, 而 L-Arg和L-His对其影响较小(图2A-D)。原因如下: 对于纯 $\mathrm{NaDC}$ 体系而言, 随着 $\mathrm{NaDC}$ 浓度增 加, $\mathrm{NaDC}$ 二聚体通过疏水作用形成了初级胶束, 在氢键作用力的驱动下初级胶束发生聚集形成二 级胶束。加入氨基酸后, $\mathrm{NaDC}$ 中的 $-\mathrm{COO}^{-}$与氨 基酸的 $-\mathrm{NH}_{3}^{+}$之间的静电作用, 以及疏水部分之 间的疏水作用使得 $\mathrm{NaDC}$ 排列得更为紧密。同时， 氨基酸分子的亲水性使其可以破坏 $\mathrm{NaDC}$ 分子周 围的水结构。此外, $\mathrm{NaDC}$ 的 $-\mathrm{OH}$ 或 $-\mathrm{COO}^{-}$与氨 基酸的 $-\mathrm{COO}^{-}$之间的氢键作用也发挥了重要作 用。氨基酸分子在 $\mathrm{NaDC}$ 初级聚集体之间的桥梁作 用有利于 $\mathrm{NaDC}$ 聚集体的进一步形成, 其相互作用 示意图如图2E所示。

此外, Mille等 22 综述了表面活性剂和氨基酸 在气/液界面上的相互作用, 从理论上论述了气/ 液界面上吸附层的溶胀及剪切流变性质, 并用来
解释相关实验现象。我们课题组研究了L-Lys、 L-Arg 和 L-His 三种碱性氨基酸对 $\mathrm{NaDC}$ 在气/液界 面上聚集行为的影响 23 。表面张力等温线及界面扩 张流变性的研究结果表明, 三种氨基酸的加入均 增大了 $\mathrm{NaDC}$ 溶液的表面张力和绝对模量, 其中 L-Lys提高能力最强。这是由于三种碱性氨基酸在 中性条件下带正电, 而 $\mathrm{NaDC}$ 作为一种阴离子型表 面活性剂带负电, $\mathrm{NaDC}$ 所含的 $-\mathrm{COO}^{-}$与氨基酸 所含的 $-\mathrm{NH}_{3}^{+}$之间的静电作用使得 $\mathrm{NaDC}$ 分子在 气/液界面上的排布较为密集，其相互作用机理图 如图2F所示。此外, 氨基酸具有一定的疏水性, 这 使其可以吸附在气/液界面上, 并且插入 $\mathrm{NaDC}$ 分子 所形成的界面层中。在静电力和疏水力的协同作 用下, L-Lys和NaDC之间的相互作用最强烈, 提 高 $\mathrm{NaDC}$ 的表面活性和绝对模量的能力最强。

\section{3 胆酸盐参与形成超分子凝胶}

近年来，自组装小分子水凝胶在化妆品、生 物材料、传感器、药物传输、刺激响应材料和制 药配方等方面的应用得到了广泛的关注度 ${ }^{24-26}$, 其 中很多水凝胶是由生物相容性的片段组成, 如氨 基酸衍生物 ${ }^{27}$ 、胆酸衍生物 ${ }^{28}$ 、碳水化合物 ${ }^{29}$ 、肽 ${ }^{30}$ 等, 因此可以应用到生物医学领域中 ${ }^{31}$ 。特别是, 大量研究表明, 属于胆酸衍生物的胆汁以及胆酸 盐, 如胆酸钠、脱氧胆酸钠、石胆酸钠等胆酸盐 因为拥有比烷烃表面活性剂复杂得多的甾体骨架 的特殊双亲结构在范德华力、氢键和疏水作用驱 使下水中能自组装形成凝胶, 且该凝胶与化学交 联而成的凝胶有很大的区别 ${ }^{32-34}$ 。

加拿大的Zhu课题组 ${ }^{35}$ 将胆酸二聚物和甲酸以 $1: 1$ 的摩尔比形成的混合物视为有机盐, 并在水溶 液中自行组装成单分散纳米纤维。纳米纤维在低 浓度的混合物中呈单分散状态，但在高浓度时相 互纠缠而形成分散性良好且随机排列的三维纤维 

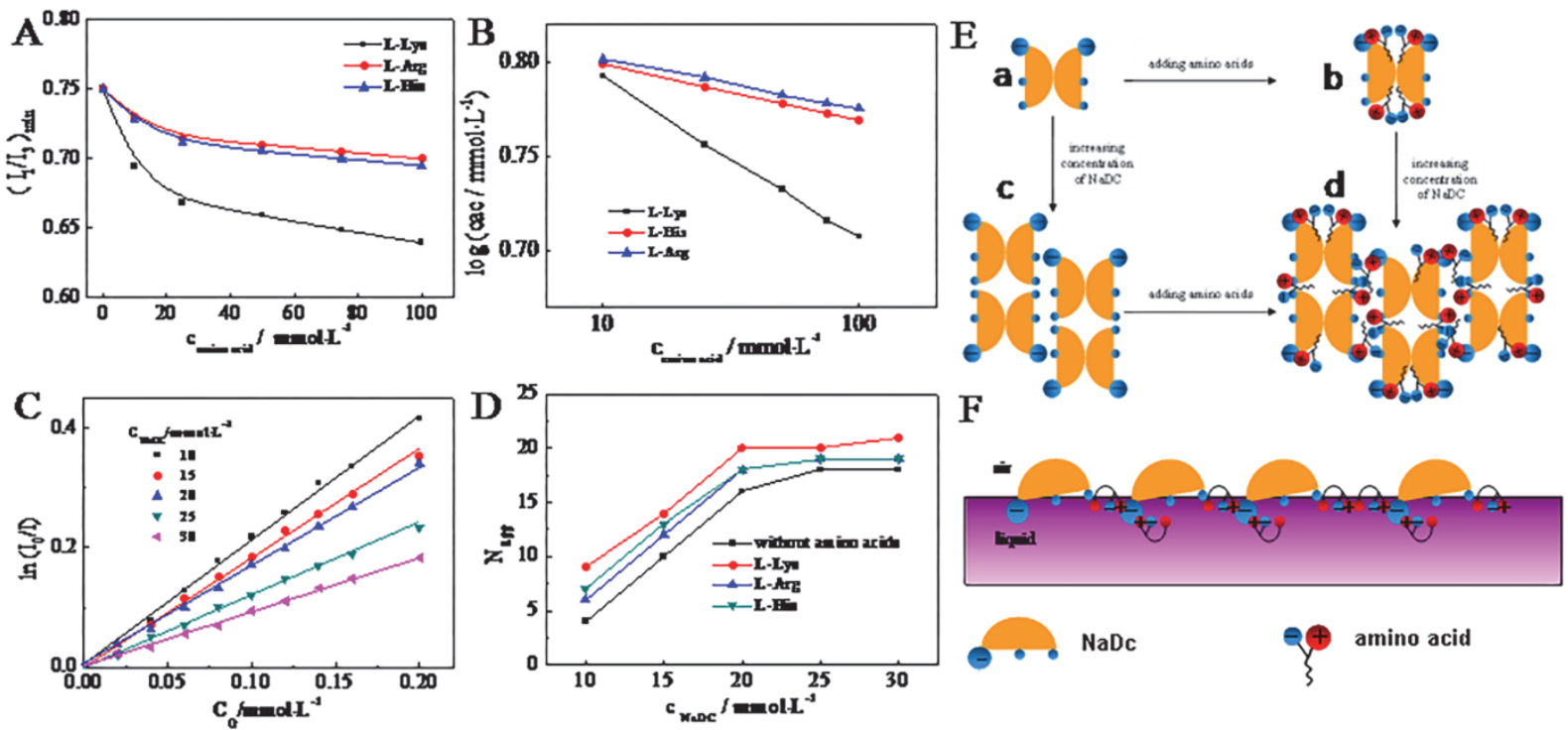

图 2 氨基酸的浓度对溶液中 $\mathrm{NaDC}$ 的(A)微极性, (B) cac 值, (C)聚集数的影响及(D)氨基酸种类对聚集数的影响。

(E)含有氨基酸时 NaDC 在溶液中的聚集行为示意图 21。(F)含有氨基酸时 NaDC 在气/液界面上的聚集示意图 ${ }^{23}$

Fig. 2 The concentration of amino acids affects the (A) micropolarity , (B) cac value, (C) aggregation number and

(D) amino acid type on aggregation number of NaDC in solution. (E) The aggregation behavior of NaDC in solution when containing amino acids ${ }^{21}$. (F) Schematic diagram of NaDC aggregation at the gas/liquid interface when containing amino acids ${ }^{23}$.

网络。当胆酸盐二聚体浓度大于凝胶点时, 纤维 网络的强度足以使水溶液形成凝胶。从低浓度的 二聚盐中获得的水凝胶是各向同性的, 在偏光片
下没有纹理出现。而当增加二聚盐浓度或在物理 搅拌作用下时, 它们则变成了各向异性(图3A)。由 此推测这种向列水凝胶可能是由来自于组成纤维

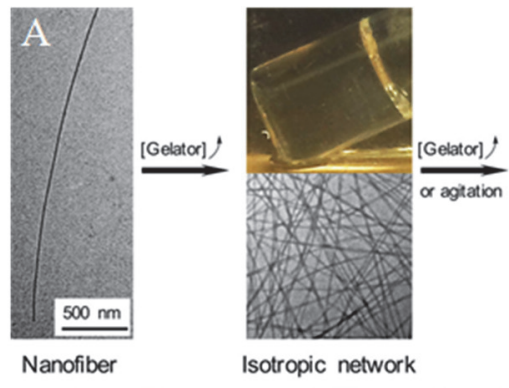

C

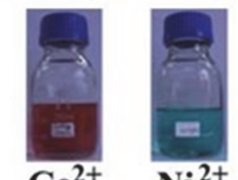

$\mathrm{Co}^{2+}$
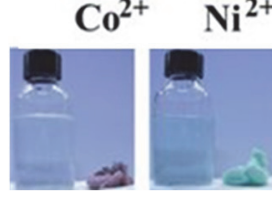

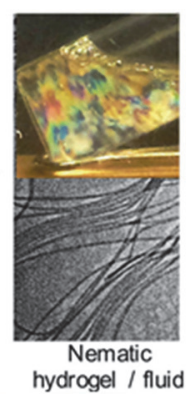

B
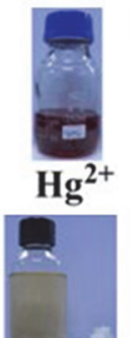

hydrogel / fluid

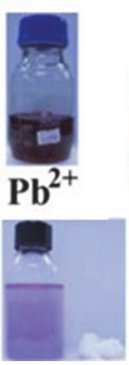

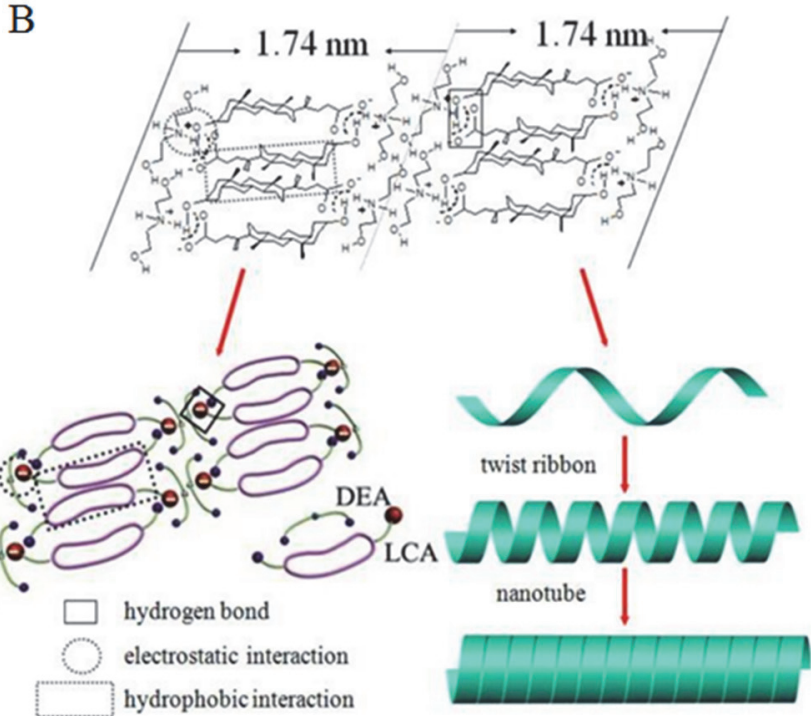

图 3 (A)胆酸二聚物与甲酸混合物在水溶液自组装形成凝胶及其偏光行为 ${ }^{35}$; (B)石胆酸/二乙醇胺自组装形成纳米管 结构的示意图, (C)不同金属离子的水溶液及石胆酸/二乙醇胺干凝胶吸附后的不同种类金属离子的水溶液样品直观图 37

Fig. 3 (A) The gelatinization and polarization behavior of the choleate dimer and formic acid mixture when it self-assembled in aqueous solution ${ }^{35}$. (B) Schematic diagram of the formation of nanotube structures by the self-assembly of stearic acid/diethanolamine, (C) Histogram of water solution samples with different metal ions and different types of metal ions after adsorption by dry gel of stacholine/diethanolamine ${ }^{37}$. 
网络的不定向纳米纤维的平行排列而形成的。

$\mathrm{Pal}$ 等 36 研究发现, 石胆酸能够与有机胺类分 子在水溶液中形成凝胶, 并且改变有机胺分子的 结构可以调控其聚集行为。山东大学郝京城课题 组 ${ }^{37}$ 也对比研究了石胆酸和三种不同种类的胺, 乙 醇胺、二乙醇胺、三乙醇胺的自组装行为, 结果 表明混合体系中氢键、静电作用、范德华作用和 疏水作用导致了不同种类的聚集体结构的形成。这 类凝胶的形成机理有相似之处, 石胆酸的羧酸根 与另一石胆酸的着基通过氢键相连, 同时与胺分 子的氮原子之间形成氢键, 将二聚石胆酸结构连 接在一起, 在空间上延伸, 形成层状结构(图3B)。 尤其是, 石胆酸和二乙醇胺可以组装得到纳米管 状结构的水凝胶, 并且由于凝胶因子上有较多的 羧酸, 可以和金属离子进行配位作用, 该凝胶在 吸附重金属离子方面有很好的效果(图3C)。

\section{1 胆酸盐/氨基酸复合凝胶}

氨基酸和胆酸盐的相互作用除了体现在稀水 溶液中的聚集行为之外, 其两者相互作用也可以 形成水凝胶, 并在药物释放和生物传感器领域具 有重要的潜在应用价值。我们选择多种氨基酸制 备了 $\mathrm{NaDC} /$ 氨基酸/ $\mathrm{NaCl}$ 凝胶, 同时研究 $\mathrm{pH} 、 \mathrm{NaCl}$ 浓度和氨基酸种类对该体系宏观与微观结构的影 响 ${ }^{38}$ 。结果表明, $\mathrm{pH}$ 降低使凝胶的机械强度增强, 原因是 $\mathrm{pH}$ 降低抑制了羧基的中和作用从而使氢键
作用增强, 而 $\mathrm{NaDC}$ 分子极性面之间的静电斥力减 弱。与L-Arg和L-Lys破坏凝胶的现象相反, 丙氨 酸和甘氨酸的加入却使 $\mathrm{NaDC}$ 凝胶的强度增强, 这 是由于它们的小尺寸及亲水性赋予了其在 $\mathrm{NaDC}$ 分子间创造更多连接的可能性。在 $\mathrm{NaDC}$ 体系中引 入 $\mathrm{NaCl}$ 可以诱导凝胶分子的聚集, 从而有利于更 宽更厚的纳米纤维组成的网络结构的形成。可以 看出, $\mathrm{NaDC}$ 凝胶对某些特殊的氨基酸和 $\mathrm{NaCl}$ 的响 应性 (图4A) 使其对包覆在凝胶中药物具有可控释 放能力, 预示了进一步应用于制药及药物释放的 潜力。我们发现在无机盐 $(\mathrm{NaCl}$ 和 $\mathrm{NaBr}$ ) 存在的情 况下, 制备出的 NaDC凝胶在加入L-Arg和L-Lys两 种氨基酸时, 表现出凝胶转变为溶液的行为 ${ }^{39}$ 。各 类表征结果证明, 凝胶是由相互交联和缠绕的纤 维构成(图4B), 这些纤维是由复杂的共价相互作 用, 尤其是氢键作用驱动形成 $\mathrm{NaDC}$ 的巨大圆形聚 集体组成的。流变实验表明 ${ }^{40}$, 卤盐 $(\mathrm{NaCl}$ 和 $\mathrm{NaBr})$ 的存在能增强凝胶的形成, 然而加入氨基酸(赖氨 酸和精氨酸)能破坏氢键从而减弱凝胶强度。原因 是加入氨基酸后, $\mathrm{NaDC}$ 周围的水结构被严重破 坏, 这对形成小的 $\mathrm{NaDC}$ 聚集体有利, $\mathrm{O}-\mathrm{H}$ 的远 程耦合振动也会受到影响。氨基酸带电电荷越 多, 亲水性越强, 破坏水结构的能力也就越强。此 外, 我们具体研究了氨基酸诱导的 $\mathrm{NaDC} /$ 无机盐 形成的超分子水凝胶的溶胀溶解过程, 以及包覆

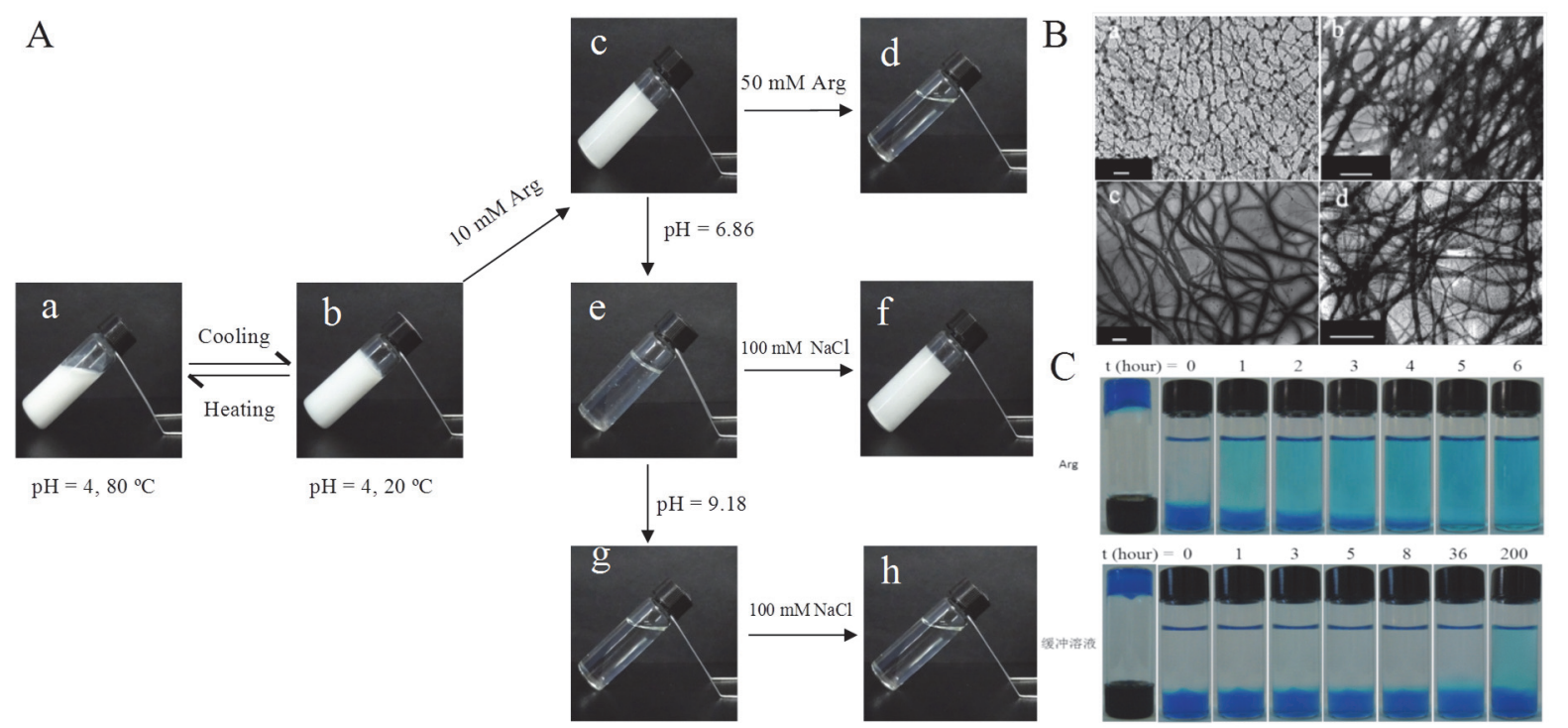

图 4 (A) $\mathrm{NaDC} /$ 氨基酸 $/ \mathrm{NaCl}$ 体系的多重刺激响应性 ${ }^{38}$; (B) $50 \mathrm{mmol} \cdot \mathrm{L}^{-1} \mathrm{NaDC}$ (a), $50 \mathrm{mmol} \cdot \mathrm{L}^{-1} \mathrm{NaDC} / 200 \mathrm{mmol} \cdot \mathrm{L}^{-1}$ $\mathrm{NaBr}$ (b), $50 \mathrm{mmol} \cdot \mathrm{L}^{-1} \mathrm{NaDC} / 10 \mathrm{mmol} \cdot \mathrm{L}^{-1} \mathrm{~L}-\mathrm{Arg}(\mathrm{c}), 50 \mathrm{mmol} \cdot \mathrm{L}^{-1} \mathrm{NaDC} / 200 \mathrm{mmol} \cdot \mathrm{L}^{-1} \mathrm{NaBr} / 10 \mathrm{mmol} \cdot \mathrm{L}^{-1}$ L-Arg (d)的 TEM 图像; (C) NaDC 凝胶对亚甲基蓝染料溶液的释放照片 ${ }^{39}$

Fig. 4 (A) Multiple stimulus response of $\mathrm{NaDC} / \mathrm{amino}$ acid $/ \mathrm{NaCl}$ system ${ }^{38}$. (B) $\mathrm{TEM}$ images of (a) $50 \mathrm{mmol} \cdot \mathrm{L}^{-1} \mathrm{NaDC}$, (b) $50 \mathrm{mmol} \cdot \mathrm{L}^{-1} \mathrm{NaDC} / 200 \mathrm{mmol} \cdot \mathrm{L}^{-1} \mathrm{NaBr}$, (c) $50 \mathrm{mmol} \cdot \mathrm{L}^{-1} \mathrm{NaDC} / 10 \mathrm{mmol} \cdot \mathrm{L}^{-1} \mathrm{~L}-\mathrm{Arg}$, (d) $50 \mathrm{mmol} \cdot \mathrm{L}^{-1} \mathrm{NaDC} / 200$ $\mathrm{mmol} \cdot \mathrm{L}^{-1} \mathrm{NaBr} / 10 \mathrm{mmol} \cdot \mathrm{L}^{-1} \mathrm{~L}$-Arg. (C) Photos of the release of NaDC gel to methylene blue dye solution ${ }^{39}$. 
在凝胶中染料(例如亚甲基蓝)的释放实验(图4C)。 结果发现, 加入赖氨酸溶液会使染料快速释放, 而磷酸缓冲溶液则没有产生类似效果。

\section{2 胆酸盐/稀土盐类复合凝胶}

近年来, 许多研究证明, 将金属离子引入到 胆酸盐溶液中可以驱动超分子聚集体的形成 ${ }^{41-45}$ 。 特别地, 三价镧系离子由于具有尖锐、环境独立、 长寿命的光致发光特性而使其在光学器件、传感、 人工拍照系统等领域显示出广泛的适用性 ${ }^{46-49}$ 。因 此, 将镧系元素引入超分子凝胶能够产生新功能 材料 50 。
Maitra等 ${ }^{51}$ 基于自组装策略使用胆酸钠和醋 酸铕制备出一种含铕水凝胶, 并发现凝胶过程中 疏水的发色团与疏水的凝胶纤维相结合，使能量 转移效率提高了几倍, 掺杂微量萠 $\left(10^{-6} \mathrm{~mol} \cdot \mathrm{L}^{-1}\right)$ 就能使镧系元素的发光显著增强的实验现象也有 力地证实了这一点。Laishram ${ }^{52}$ 发现 $\mathrm{Eu}^{3+}$ 与胆酸在 甲醇中能够形成凝胶, 并与在水溶液中形成的 $\mathrm{Eu}^{3+} /$ 胆酸水凝胶进行比较研究(图 $5 \mathrm{~A}$ )。我们知道, 水是 $\mathrm{Eu}^{3+}$ 苂光的常见猝灭剂, 其猝灭率比 $\mathrm{MeOH}$ 高。因此，在 $\mathrm{Eu}^{3+}$ 衍生的发光材料中，由甲醇代 替水应该会使 $\mathrm{Eu}^{3+}$ 发光增强。然而，在 $\mathrm{Eu}^{3+} /$ 胆酸
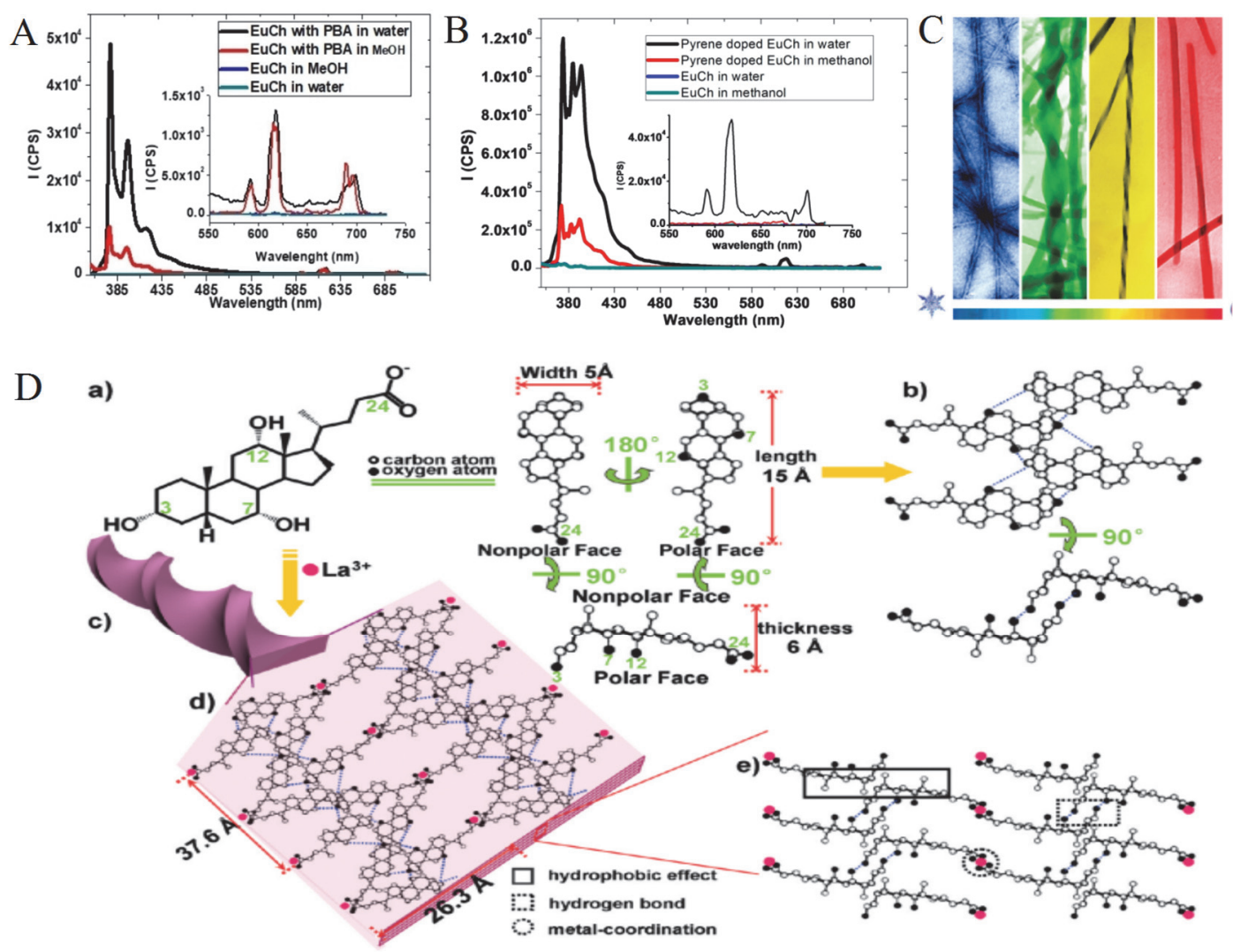

图 5 (A) $\mathbf{E u}^{3+}$ /胆酸在水中和甲醇中形成的凝胶在萠存在或不存在情况下的发射光谱 $\left(\lambda_{\mathrm{ex}}=336 \mathrm{~nm} ; \mathbf{E u}^{3+}-5 \mathrm{mmol} \cdot \mathrm{L}^{-1}\right.$, 胆酸 $-15 \mathrm{mmol} \cdot \mathrm{L}^{-1}$, 萠 $-5 \mathrm{mmol} \cdot \mathrm{L}^{-1}$; 内嵌图为 $\mathrm{Eu}^{3+}$ 的发射谱图 ); (B) $\mathrm{Eu}^{3+} /$ 胆酸的甲醇凝胶与水凝胶 $\left(5 / 15 \mathrm{mmol} \cdot \mathrm{L}^{-1}\right)$ 中 $\mathrm{Eu}^{3+}$ 在 1-萠丁酸 $\left(12.5 \mathrm{mmol} \cdot \mathrm{L}^{-1}\right)$ 存在下的敏化发光 ${ }^{52}$; (C) 镧离子/胆酸超分子凝胶随温度变化发生的微观 结构变化, (D)钵离子与胆酸温度响应性自组装的机理示意图, (a)胆酸的分子结构, (b)双分子层型胆酸 主体结构的分子聚集方式, (c)螺旋纳米带, 分子模型的(d)俯视和(e)侧视图 53

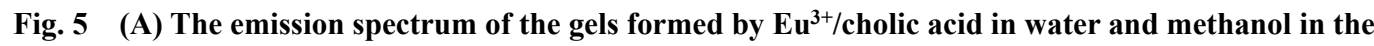
presence or absence of pyrene $\left(\lambda_{\mathrm{ex}}=336 \mathrm{~nm} ; \mathrm{Eu}^{3+}-5 \mathrm{mmol} \cdot \mathrm{L}^{-1}\right.$, bile acid-15 $\mathrm{mmol} \cdot \mathrm{L}^{-1}$, pyrene-5 $\mathrm{mmol} \cdot \mathrm{L}^{-1}$; the embedded picture shows the emission spectrum of $\mathrm{Eu}^{3+}$ ), (B) The sensitized luminescence $\mathrm{Eu}^{3+} / \mathrm{cholic}$ acid methanol gel $\left(5 / 15 \mathrm{mmol} \cdot \mathrm{L}^{-1}\right)$ and hydrogel in the presence of 1-pyrene butanic acid $\left(12.5 \mathrm{mmol} \cdot \mathrm{L}^{-1}\right)^{52}$. (C) The microstructures change of lanthanum ion/cholic acid supermolecular gel at different temperatures, (D) mechanism diagram of temperature-responsive self- assembly of lanthanum ion and bile acid, (a) Molecular structure of cholate, (b) molecular aggregate of a bilayer type of cholate host framework (the blue dotted lines denote H-bonds), (c) twisted nanoribbon, (d) top view, and (e) cross-sectional view of the molecular model ${ }^{53}$. 
盐水凝胶中观察到显著的萠-介导的 $\mathrm{Eu}^{3+}$ 的致敏发 光, 而在甲醇凝胶中却没有荧光(图5B)。为了合理 解释这一悖论, 该课题组进行了一系列实验, 最终 得出原因, 在甲醇凝胶中萠远离凝胶纤维, 从而 与 $\mathrm{Eu}^{3+}$ 之间没有能量传递, 也就无法使 $\mathrm{Eu}^{3+}$ 敏化发 光。而在水凝胶中, 疏水作用使萠与纤维相连, 从 而与能够将能量传递给 $\mathrm{Eu}^{3+}$ 。因此, 水对 $\mathrm{Eu}^{3+} /$ 胆 酸水凝胶中 $\mathrm{Eu}^{3+}$ 的苂光增强起到了间接但重要的 积极作用。

黄建滨课题组 ${ }^{53}$ 通过镧离子与胆酸进行超分 子自组装得到了具有一维螺旋状结构的发光凝 胶。作用力分析结果表明该自组装过程的驱动力 主要是针离子与羧基的配位作用。有趣的是, 他 们还发现该凝胶的微观结构随温度的逐渐升高经 历了由纳米管到螺旋纳米线及纳米带的转变 (图 $5 \mathrm{C})$ 。这种独特的温度效应被认为是胆酸中羧基的 作用。如图5D所示的机理图, 由于胆酸具有亲水 性, 胆酸上的羧基通过氢键与水分子间发生相互 作用。当温度升高时, 氢键作用减弱, 导致亲水 性降低, 这被认为是镧离子/胆酸之间的自组装具 有温度响应性的结构性原因。得益于降低的亲水 性, 镧离子/胆酸聚集体的形成得到了促进, 因此 高温有利于大聚集体的形成。

我们课题组以 $\mathrm{NaDC}$ 和 $\mathrm{Eu}\left(\mathrm{NO}_{3}\right)_{3}$ 为原料通过 超分子自组装方法也制备出发光凝胶。实验结果
表明, 该超分子凝胶具有优异的成胶能力与机械 强度, 成胶的主要驱动力是疏水作用、氢键作用、 金属配位作用、空间效应以及范德华力 ${ }^{54}$ 。该凝胶 的微观结构是由发红色荧光的三维球状聚集体构 成的, 同时所获得发光凝胶具有优异的成胶能力 和机械性能。荧光光谱结果表明荧光最大发射峰 位置出现在 $\mathrm{Eu}\left(\mathrm{NO}_{3}\right)_{3}$ 和 $\mathrm{NaDC}$ 化学计量比为 $1: 3$ 的时候, 这是由于在此比例下销被周围 $\mathrm{COO}^{-}$有效 配位率最大的结果。

\section{3 胆酸盐/氧化石墨烯复合凝胶}

虽然胆酸盐在溶液中和其他小分子物质相互 作用可以自发形成水凝胶, 但这类胆酸盐水凝胶 的粘弹性和机械性质并不强, 因此我们通常需要 添加其它材料来形成复合水凝胶以增强其强度和 应用范围。有研究表明, 添加有机/无机填料可以在 很大程度上增强水凝胶的粘弹性和机械性能 55,56 。 其中, 氧化石墨烯 $(\mathrm{GO})$ 可以作为一种纳米级填料 添加到水凝胶体系中形成石墨烯基水凝胶体系从 而提高水凝胶的强度 ${ }^{57-61}$ 。

我们课题组系统研究了在不同浓度缓冲液中 $\mathrm{GO}$ 对 $\mathrm{NaDC}$ 水凝胶的性质影响, 并探讨了复合水 凝胶的染料吸附能力 ${ }^{62}$ 。实验结果表明, GO 被很 好地分散到 $\mathrm{NaDC}$ 凝胶中, 并且随着 $\mathrm{GO}$ 的加入 $\mathrm{NaDC} / \mathrm{GO} / \mathrm{PBS}$ 水凝胶的机械强度增强。此外, 含 有 $\mathrm{GO}$ 的复合水凝胶对亚甲基蓝溶液有较强的吸
A

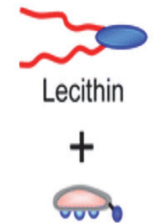

Bile Salts$$
\text { C }
$$

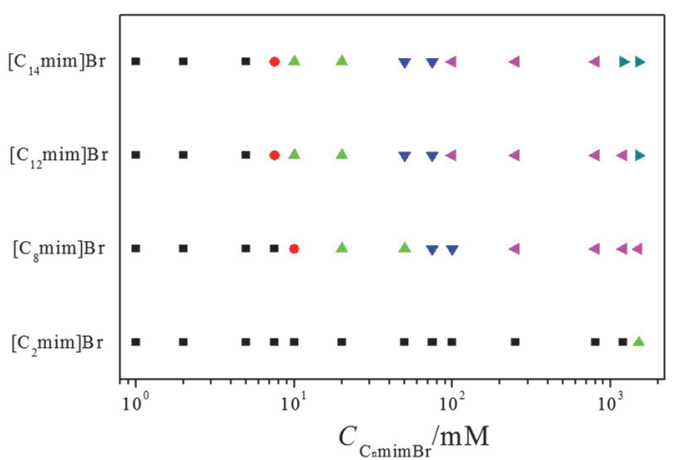

B

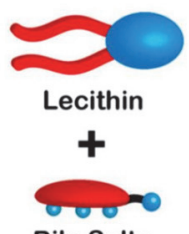

Bile Salts

D
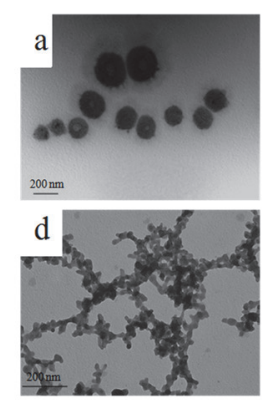

Hydrogels
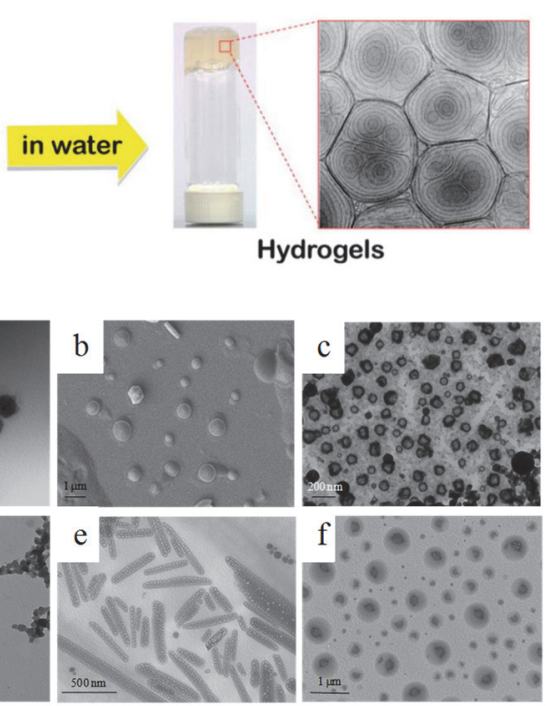

图 6 胆酸盐与卵磷脂自组装形成的(A)蠕虫状胶束 ${ }^{65}$ 和(B)多层囊泡构筑的水凝胶 ${ }^{66}$; (C) NaDC 和不同链长的 咪唑类离子液体 $\left[\mathrm{C}_{n} \mathrm{mim}\right] \mathrm{Br}(n=2,8,12,14)$ 混合体系在 $20^{\circ} \mathrm{C}$ 放置 4 周后的平衡相图及(D)不同样品的形貌 ${ }^{67}$

Fig. 6 (A) worm-like micelle ${ }^{65}$ and (B) multilayer vesicles hydrogels ${ }^{66}$ formed by the self-assembly of cholate and lecithin. (C) The equilibrium phase diagram of $\mathrm{NaDC}$ and imidazole liquids $\left[\mathrm{C}_{n} \mathrm{mim}\right] \mathrm{Br}(n=2,8,12,14)$ mixtures with different chain lengths at $20{ }^{\circ} \mathrm{C}$ after placement of 4 weeks and (D) morphology of different samples ${ }^{67}$. 
附作用, 这是由于 $\mathrm{GO}$ 片层上含有大量的含氧官能 团, 这些官能团可以和很多有机分子相互作用。在 染料吸附的过程中复合水凝胶没有发生溶胀溶解 现象, 而是保持着凝胶的状态。然而不含有 $\mathrm{GO}$ 的 纯 $\mathrm{NaDC}$ 水凝胶则发生了溶胀溶解现象, 不具有 染料吸附能力。 $\mathrm{GO}$ 的加入提高了脱氧胆酸钠成 胶能力, $\mathrm{GO}$ 在该体系中起到了物理交联剂的作 用, 同时 $\mathrm{GO}$ 上的含氧官能团和 $\mathrm{NaDC}$ 之间发生氢 键作用。

\section{4 胆酸盐/表面活性剂凝胶}

阴阳离子表面活性剂复配体系由于结合了两 种表面活性剂的物理化学性质, 通常具有丰富的 相行为及聚集体形貌变化, 如球形胶束, 蠕虫状 胶束、囊泡、凝胶、沉淀等63,64。Tung等65使用胆 酸盐与卵磷脂这两种带相反电荷的表面活性剂的 甲醇混合液中加入去离子水和电解质, 得到了高 粘度的蠕虫状胶束(图6A)。随后该课题组又调节 二者比例制备了由多层囊泡构成的复合凝胶 (图 $6 \mathrm{~B})$ 。据此, 研究者发现通过调节胆酸钠/卵磷脂两 种物质摩尔比, 可以得到粘弹性流体、半凝胶等 丰富的相行为 ${ }^{66}$ 。

我们课题组研究了在中性条件下 (PBS, $\mathrm{pH}=$ 7) $\mathrm{NaDC}$ 与咪唑类离子液体 $\left[\mathrm{C}_{n} \operatorname{mim}\right] \mathrm{Br}(n=2,8$,
12，14)之间的相互作用。结果发现, 改变离子液 体的链长由 $14 、 12$ 减小到 8 , 再减小到 2 , 依次得 到囊泡、两相、凝胶相(图6C)。而且, 链长大于 8 的咪唑离子液体也会破坏 $\mathrm{NaDC}$ 凝胶, 发生溶胶、 沉淀、两相、溶液、液晶一系列相态变化 ${ }^{67}$ 。然而 短链的离子液体 $\left[\mathrm{C}_{2} \mathrm{mim}\right] \mathrm{Br}$ 则增强了 $\mathrm{NaDC}$ 凝胶的 机械性能, 并且可以诱导凝胶体系形成微晶。在 整个 $\mathrm{NaDC} /\left[\mathrm{C}_{n} \mathrm{mim}\right] \mathrm{Br}$ 自组装过程中, 静电作用、 疏水作用、分子空间构型均是影响自组装相行为 变化及微观结构的主要因素, 所得样品的形貌也 有所差别(图6D)。

\section{4 胆酸盐参与构筑的微纳米材料}

\section{1 表面活性剂在自组装制备微纳米材料中的} 作用

自组装技术做为一种简单而高效的方法 ${ }^{68,69}$, 目前已经被广泛应用于微纳米材料的制备, 主要 方法有层层自组装 ${ }^{70-73}$ 、相转移自组装 ${ }^{74,75}$ 以及超 分子自组装76-78 以及胶体晶体自组装等等。表面活 性剂的两亲性及其在溶液中丰富的聚集行为使其 成为自组装中常用的模板和构筑基元, 包括与高 分子 79,80 、无机分子 81,82 及有机分子 83,84 等的相互作 用。由于纳米材料具有巨大的比表面积和较高的

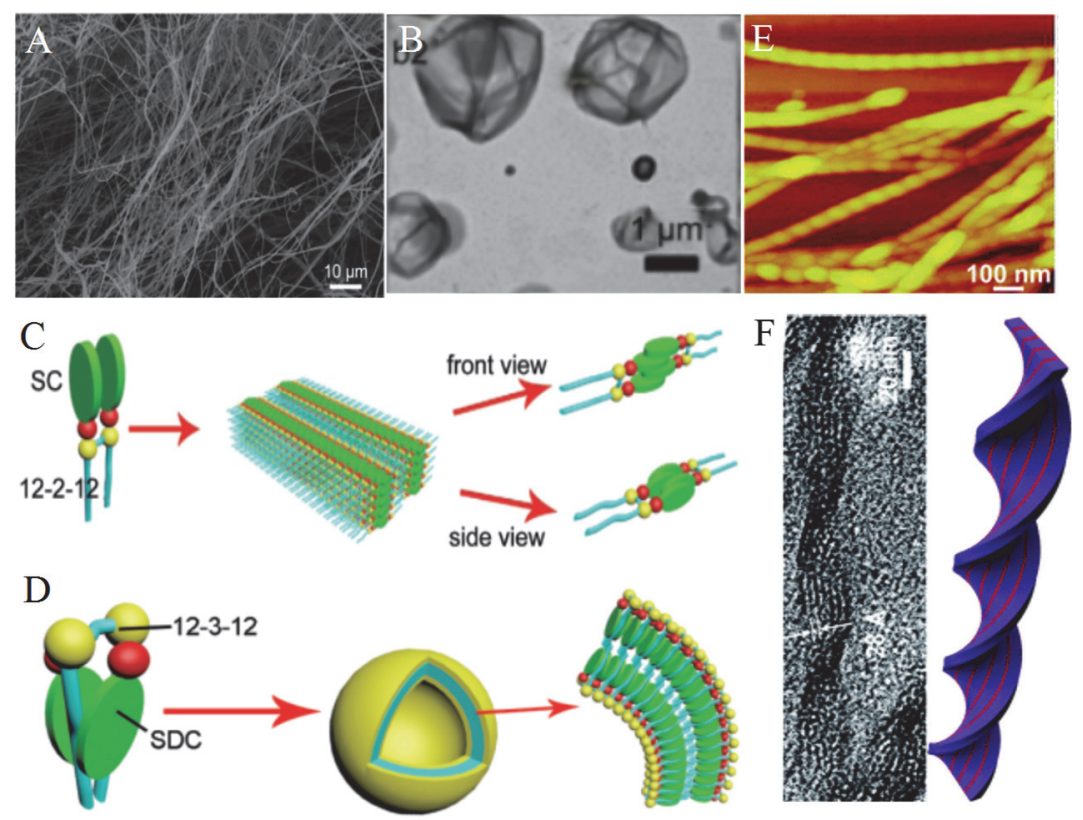

图 7 (A) 12-2-12/SC 形成的纳米纤维状聚集体的 SEM 图像; (B) 12-3-12/SDC 形成的囊泡聚集体的 TEM 图像; (C)纳米纤维和(D)囊泡形成机理图 ${ }^{88}$; 谷胱甘肽和脱氧胆酸钠自组装形成的超长螺旋纳米线的(E) AFM 图像, 和(F)高分辨 TEM 图像及示意图 89

Fig. 7 (A) SEM images of nanofibrous aggregates formed by12-2-12/SC. (B) TEM images of vesicular aggregates formed by 12-3-12/SDC. The formation mechanisms of (C) nanofibers and (D) vesicle ${ }^{88}$. (E) AFM images, (F) high-resolution TEM images and schematic of glutathione and sodium deoxycholic acid self-assembled ultra-long spiral nanowires ${ }^{89}$. 
表面能, 其发生团聚的倾向非常大。在纳米材料 合成过程中加入表面活性剂, 不仅可在初期作为 模板剂, 而且能在刚形成的纳米晶种表面快速吸 附, 从而有效防止材料的团聚。表面活性剂在纳 米材料合成过程中的稳定作用是通过表面活性剂 吸附在纳米材料表面, 利用静电排斥、空间位阻 与范德华力之间的竞争达到平衡而实现的。例如, Geoffrey课题组 ${ }^{85}$ 利用表面活性剂 $\mathrm{TMA}_{4} \mathrm{Ge}_{4} \mathrm{~S}_{10}$ 作 为模板与环状的多金属氧簇 $\{$ Mo154 $\}$ 之间的相互 的静电作用成功制备了有机无机杂化囊泡。该囊 泡具有优异的光电性质。我们课题组曾分别研究 了多金属氧酸盐 $\mathrm{Na}_{9}\left[\mathrm{EuW}_{10} \mathrm{O}_{36}\right] \cdot 32 \mathrm{H}_{2} \mathrm{O}$ 与多巴胺 ${ }^{86}$ 和烷基胺 ${ }^{87}$ 两种两亲分子的自组装行为, 均得到了 纳米花结构。

\section{2 胆酸盐参与的自组装法制备微纳米材料}

而胆酸盐作为一类生物表面活性剂也常常被 用来辅助合成微纳米材料。陈晓课题组 ${ }^{88}$ 利用 Gemini表面活性剂与胆酸盐之间的静电作用构建 了超分子复合物。实验结果表明, 通过改变表面
活性剂的烷基链长和间隔基团的长度, 以及胆酸 盐胆甾环骨架上羟基的数目和位置, 可以使聚集 体的形貌发生显著的变化, 分别得到纳米纤维和 囊泡状超分子聚集体(图7A, B)。同时发现聚集体 的形成是静电相互作用, 疏水作用及氢键的协同 作用的结果 (图7C, D)。黄建滨课题组 ${ }^{89}$ 胆酸钠与 金属离子自组制备了超长的螺旋纳米线(图7E, F), 该过程是氢键、配位作用、和疏水效应等多种作 用力协同效的结果。随后利用该金属-胆酸超分子 系统设计了溶胶-凝胶和模板自组装两种策略, 分 别合成了 $\mathrm{SiO}_{2}$ 和 $\mathrm{ZnS}$ 螺旋纳米材料。我们设计了一 种简便有效的模板自组装策略, 以铜离子为无机 组分, 胆酸钠为有机组分合成了三维花状 $\mathrm{Cu}_{3}\left(\mathrm{PO}_{4}\right)_{2} \cdot 3 \mathrm{H}_{2} \mathrm{O}$ 纳米结构 ${ }^{90}$ 。实验结果表明该纳米 花对降解阳离子染料表现出显著的催化活性以 及较高的稳定性。此外, 在循环催化亚甲基蓝的 实验中, 纳米花至少可以循环使用六次, 表明其 在废水处理方面的应用潜力。

染料一般具有廉价易得、功能基团多样化、
A
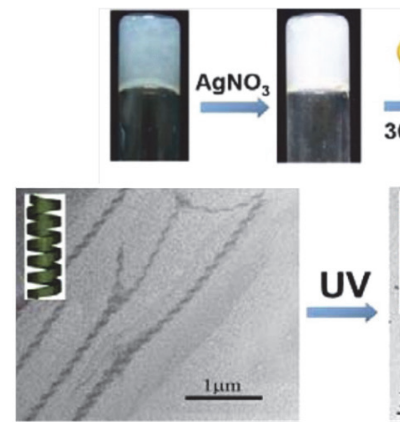

$\mathrm{C}_{\text {a }}$
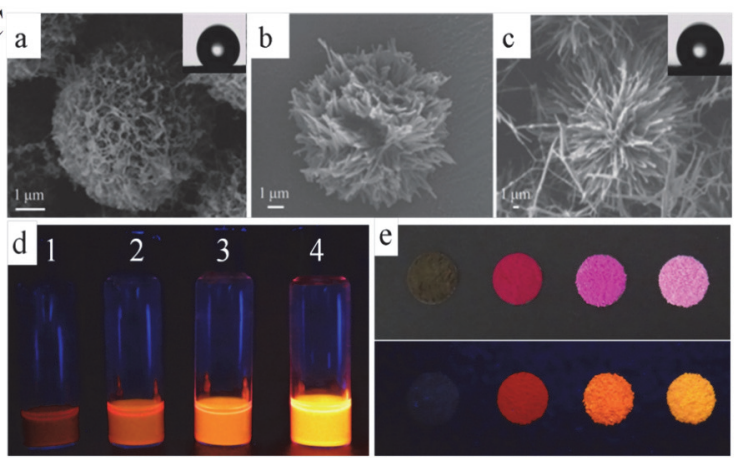

B
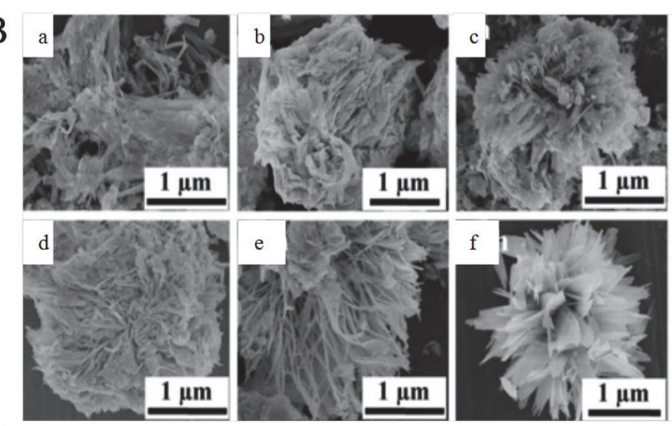

$\mathrm{D}$

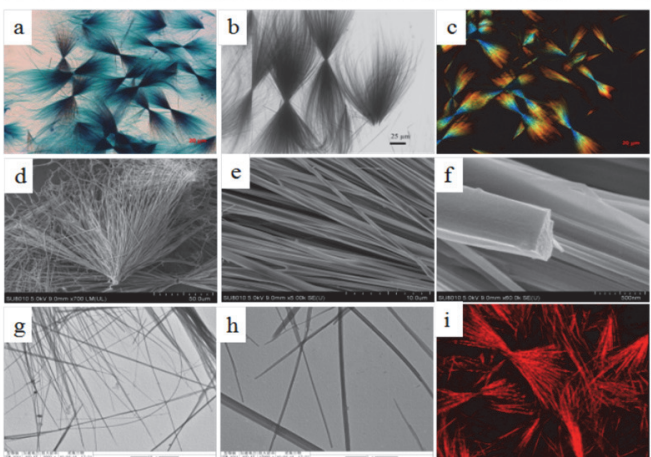

图 8 (A) 以 $\mathrm{GSH} / \mathrm{NaDC}$ 凝胶为模板合成 $\mathrm{Ag}$ 纳米粒子 ${ }^{91}$; (B) $\mathrm{SC}$ 辅助合成的 $\mathrm{CdWO}_{4}$ 纳米花随时间变化的生长过程 ${ }^{22}$,

(a) $1 \mathrm{~h}$, (b) $3 \mathrm{~h}$, (c) $6 \mathrm{~h}$, (d) $9 \mathrm{~h}$, (e) $12 \mathrm{~h}$, (f) $24 \mathrm{~h}$; (C) NaDC/Rh 自组装形成的(a)微球结构和(b, c)海胆状结构及 (d) 上层溶液和(e)沉淀的苂光照片 ${ }^{93}$; (D) SC/MB 自组装形成的超长纤维的 $(a, b)$ 光学显微镜照片,

(c)偏光显微镜照片, (d-f) SEM、(g, h) TEM 和(i)激光共聚焦图像 96

Fig. 8 (A) Ag nanoparticles were synthesized using GSH/NaDC gel as a template ${ }^{91}$. (B) Growth process of $\mathrm{CdWO}_{4}$ nanoflowers over time based on SC-assisted synthesis ${ }^{92}$, (a) $1 \mathrm{~h}$, (b) $3 \mathrm{~h}$, (c) $6 \mathrm{~h}$, (d) $9 \mathrm{~h}$, (e) $12 \mathrm{~h}$, (f) $24 \mathrm{~h}$.

(C) The (a) microsphere structure and(b, c) sea urchin structure formed by $\mathrm{NaDC} / \mathrm{Rh}$ self-assembly and fluorescence photographs of (d) the upper solution and (e) the precipitates ${ }^{93}$. (D) Overlength fibres formed by SC/RMB self-assembly,

(a, b) Optical microscopy images, (c) POM image, (d-f) SEM images, (g, h) TEM images and (i) CLSM images ${ }^{96}$. 
分子形状规则、具有多重作用力等特点, 并且很 多具有优异的光电特性, 因此被认为是构筑功能 性微纳米材料的理想基元。表面活性剂与染料分 子间主要通过静电力、氢键、范德华力以等驱动 力来进行。在通常情况下, 染料分子与表面活性 剂分子作用后形成的复合物会使染料的溶解性、吸 收光谱和染色性能发生很大变化。

胆酸钠是最常见胆酸盐之一, 然而关于其参 与制备微纳米材料的报道比较少。例如, 郝京诚 等 ${ }^{91}$ 以谷胱甘肽 (glutathione, $\mathrm{GSH}$ ) 和脱氧胆酸钠 自组装形成凝胶, 该凝胶由超长的螺旋纳米线组 成, 随后以该螺旋的纳米线为模板UV诱导还原制 备 $\mathrm{Ag}$ 纳米粒子 (图 $8 \mathrm{~A}$ )。师进生等 92 利用胆酸钠作为 自组装前驱体, 在低温 $35{ }^{\circ} \mathrm{C}$ 条件下简单制备3D $\mathrm{CdWO}_{4}$ 纳米花结构, 并用 SEM追踪了其随时间变 化的生长过程, 如图8B所示。近几年, 我们课题 组系统研究了胆酸盐与染料的相互作用。首先, 我 们以 $\mathrm{NaDC}$ 和染料分子罗丹明 $\mathrm{B}(\mathrm{RhB})$ 为原料进行 了ISA研究 ${ }^{93}$ 。结果发现, 通过改变 $N a D C$ 和RhB两 种物质的比例可以得到不同的具有高级结构的形 貌, 如多孔的微球结构和海胆状结构(图8C, a-c)。 这种多级结构表现出良好的超疏水性能, 表现出 在超疏水材料方面有潜在的应用。此外, $\mathrm{NaDC}$ 和 $\mathrm{RhB}$ 之间的相互作用限制了染料分子内的电荷转 移, 大大削弱了其自身具有的聚集诱导猝灭性质, 增强了染料的荧光性质(图 $8 \mathrm{C}, \mathrm{d}-\mathrm{e}$ )。随后, 我们 使用 $\mathrm{NaOH}$ 将体系调节至碱性环境研究其组装行 为的变化。结果发现该体系形成了具有热致液晶 行为和固态发光性质的海胆状微晶, 将其溶于有 机溶剂, 发现改变有机溶剂的混合比例可以得到 形貌与发光性质不同的溶液, 并将其应用于 $\mathrm{Fe}^{3+}$ 的检测 94 。随后, 我们分别通过ISA策略研究了 $\mathrm{NaDC}$ 与 $\mathrm{AO}{ }^{95}$ 、胆酸钠 $(\mathrm{SC})$ 与阳离子染料 $(\mathrm{MB}){ }^{96}$ 的相互作用, 都能制备出具有固态荧光发射的复 合物。我们使用光学显微镜(图8D, a-b)、SEM(图 8D, d-f) 和TEM(图 8D, g, h) 表征了 SC/MB 的超 长纤维结构, 并证明了其具有偏光性(图8D, c) 和 苂光性质(图8D，i)。

\section{5 结语与展望}

综上所述, 胆酸盐作为一类生物表面活性剂, 不仅在生物体内起到了重要的生理调节功能, 而 且被越来越广泛地应用于超分子自组装、微纳米 材料的制备等领域。由于胆酸盐具有特殊的几何 结构与两亲性使其聚集行为非常丰富, 而生物分 子如氨基酸、无机盐、稀土金属离子、氧化石墨
烯等与胆酸盐相互作用可以显著影响其聚集行 为, 形成胶束、囊泡、凝胶等聚集结构; 同时还 可以赋予凝胶丰富的光电性质、显著提高凝胶的 机械强度等。另外, 胆酸盐在自组装制备微纳米 材料的过程中可以起到模板作用, 辅助形成形貌 可控且分散性良好的微纳米结构; 还可以作为构 筑单元与带相反电荷的分子自组装形成超分子结 构, 制备超疏水材料、苂光探针和电化学活性纳 米材料。可以看出, 虽然胆酸盐类生物表面活性 剂在超分子自组装和制备各种功能性纳米材料方 面得到了一定的发展, 然而, 目前的研究重点基 本集中于胆酸盐的模板作用和与染料的离子自组 装, 关于胆酸盐本身的生物生理方面的功能则利 用的较少, 所以对胆酸盐的功能开发仍然是一项 值得继续深入研究的课题。我们期望在以后的工 作中寻求与生物领域的合作, 将胆酸盐参与自组 装和形成微纳米材料后的功能开发拓展到生理与 生物方面, 充分发挥胆酸盐调节胆固醇代谢、增 强胰脂肪酶的活性, 促使脂肪酸和脂溶性维生素 的吸收等作用, 以期更好地展现其功能。

\section{References}

(1) Wu, T. H.; Wang, Z. N. Prog. Chem. 2011, 23 (1), 80. [吴同浩, 王仲妮. 化学进展, 2011, $23(1), 80]$

(2) Li, Y. X.; Yan, L.Y.; Liu, K.; Wang, J.; Wang, A. H.; Bai, S.; Yan, X. H. Small 2016, 12 (19), 2575. doi: 10.1002/smll.201600230

(3) Lehn, J. M. Proc. Natl. Acad. Sci. USA 2002, 99 (8), 4763. doi: 10.1073/pnas.072065599

(4) Wiedmann, T. S.; Liang, W.; Kamel, L. Pharm. Res. 2002, 19 (8), 1203. doi: 10.1023/A:1019858428449

(5) Xu, H. M.; Li, J.; Qiao, Y. F.; Zhang, M.; Zhang, H. Y.; Wang, Y. R.; Hu, P. Chem. J. Chin. Univ. 2017, 38 (7), 1148. [徐红梅, 李洁, 乔云帆, 张敏, 章弘扬, 王月荣, 胡坪. 高等学校化学学报, 2017, 38 (7), 1148.] doi: 107503/cjcu20160929

(6) Tao, K.; Makam, P.; Aizen, R.; Gazit, E. Science 2017, 358 (6365), 9756. doi: 10.1126/science.aam9756

(7) Saitoh, T.; Fukuda, T.; Tani, H.; Kamidate, T.; Watanabe, H. Anal. Sci. 1996, 12 (4), 569. doi: 10.2116/analsci.12.569

(8) Gregory P. T.; Gregory T. D.; Douglas F. C.; David P. C. J. Am. Chem. Soc. 2004, 126 (35), 11024. doi: 10.1021/ja047589c

(9) Gubitosi, M.; Travaglini, L.; di Gregorio, M. C.; Pavel, N. V.; Tato, J. V.; Sennato, S.; Olsson, U.; Schillen, K.; Galantini, L. Angew. Chem. Int. Edit. 2015, 54 (24), 7018.

doi: 10.1002/anie. 201500445

(10) Gregorio, M. C.; Pavel, N. V.; Miragaya, J.; Jover, A.; Meijide, F.; Tato, J. V.; Tellini, V. H. S.; Galantini, L. Langmuir 2013, 29 (40), 
12342. doi: $10.1021 / 1 a 402602 d$

(11) Saitoh, T.; Fukuda, T.; Tani, H.; Kamidate, T.; Watanabe, H. Anal. Sci. 1996, 12 (4), 569. doi: 10.2116/analsci.12.569

(12) Smidkova, M.; Spundova, M.; Marecek, Z.; Entlicher, G. Fund. Clin. Pharmacol. 2003, 17 (3), 331. doi: 10.1046/j.1472-8206.2003.00157.x

(13) Orioni, B.; Roversi, M.; La Mesa, C.; Asaro, F.; Pellizer, G.; D'Errico, G. J. Phys. Chem. B 2006, 110 (24), 12129. doi: $10.1021 /$ jp055950r

(14) De, S.; Das, S.; Girigoswami, A. Colloids Surf. B 2007, 54 (1), 74. doi: 10.1016/j.colsurfb.2006.09.015

(15) Yu, L.; Lu, T.; Luan, Y. X.; Liu, J.; Xu, G. Y. Colloids Surf. A 2005, 257-258, 37. doi: 10.1016/j.colsurfa.2004.10.066

(16) Small, D. M.; Penkett, S. A.; Chapman, D. Biochim. Biophys. Acta 1969, 176 (1), 178. doi: 10.1016/0005-2760(69)90086-1

(17) Carey, M. C.; Small, D. M. J. Colloid Interface Sci. 1969, 31 (3), 382. doi: 10.1016/0021-9797(69)90181-7

(18) Kawamura, H.; Murata, Y.; Yamaguchi, T.; Igimi, H.; Tanaka, M.; Sugihara, G.; Kratohvil, J. P. J. Phys. Chem. 2002, 93 (8), 3321. doi: $10.1021 / \mathrm{j} 100345 \mathrm{a} 087$

(19) D'Alagni, M.; D'Archivio, A. A.; Galantini, L.; Giglio, E. Langmuir 1997, 13 (22), 581. doi: 10.1021/la970337n

(20) Campanelli, A. R.; Sanctis, C. D. S.; Giglio, E.; Viorel Pavel, N.; Quagliata, C. J. Incl. Phenom. 1989, 7 (4), 391. doi: 10.1007/BF01079774

(21) He, F.; Xu, G. Y.; Pang, J. Y.; Han, T. T.; Liu, T.; Yang, X. D. Luminescence 2012, 27 (1), 4. doi: 10.1002/bio.1312

(22) Miller, R.; Wüstneck, R.; Krägel, J.; Kretzschmar, G. Colloids Surf. A 1996, 111 (1-2), 75. doi: 10.1016/0927-7757(95)03492-7

(23) He, F.; Xu, G. Y.; Pang, J. Y.; Ao, M. Q.; Han, T. T.; Gong, H. J. Langmuir 2011, 27 (2), 538. doi: 10.1021/la103478c

(24) Hirst, A. R.; Escuder, B.; Miravet, J. F.; Smith, D. K. Angew. Chem. Int. Ed. 2008, 47 (42), 8002. doi: 10.1002/anie.200800022

(25) Banerjee, S.; Das, R. K.; Maitra, U. J. Mater. Chem. 2009, 19 (37), 6649. doi: 10.1039/B819218A

(26) Qiao, Y.; Lin, Y. Y.; Zhang, S. F.; Huang, J. B. Chem. Eur. J. 2011, 17 (18), 5180. doi: 10.1002/chem.201003255

(27) Kiyonaka, S.; Sada, K.; Yoshimura, I.; Shinkai, S.; Kato, N.; Hamachi, I. Nat. Mater. 2003, 3(1), 58. doi: 10.1038/nmat1034

(28) Maitra, U.; Mukhopadhyay, S.; Sarkar, A.; Rao, P.; Indi, S. Angew. Chem. Int. Ed. 2001, 40 (12), 2281. doi: 10.1002/1521-3773(20010618)

(29) Jung, J. H.; Shinkai, S.; Shimizu, T. Chem. Eur. J. 2002, 8 (12), 2684. doi: 10.1002/1521-3765(20020617)

(30) Chen, L.; Revel, S.; Morris, K. C.; Serpell, L.; Adams, D. J. Langmuir 2010, 26 (16), 13466. doi: 10.1021/1a102059x
(31) Cunningham, A. J.; Robinson, M.; Banquy, X.; Leblond, J.; Zhu, X. X. Mol. Pharma. 2018, 15 (3), 1266. doi: 10.1021/acs.molpharmaceut.7b01091

(32) Le Devedec, F.; Fuentealba, D.; Strandmn, S.; Bohne, C.; Zhu, X. X. Langmuir 2012, 28 (37), 13431. doi: 10.1021/1a303218q

(33) Song, S. S.; Feng, L.; Song, A. X.; Hao, J. C. J. Phys. Chem. B 2012, 116 (42), 12850. doi: 10.1021/jp3066025

(34) Goldshleger, N. F.; Lobach, A. S.; Baulin, Vladimir. E.; Baulin, V. E. Russ. Chem. Rev. 2017, 86 (4), 269. doi: 10.1070/RCR4682

(35) Zhang, M.; Fives, C.; Waldron, K. C.; Zhu, X. X. Langmuir 2017, 33 (4), 1084. doi: 10.1021/acs.langmuir.6b04033

(36) Pal, A.; Basit, H.; Sen, S.; Aswal, V. K.; Bhattacharya, S. J. Mater. Chem. 2009, 19 (25), 4325. doi: 10.1039/b903407b

(37) Song, S. S.; Wang, H. Q.; Song, A. X.; Hao, J. C. Chem. Asian J. 2014, 9 (1), 245. doi: 10.1002 /asia.201300892

(38) Zhang, Y. J.; Xin, X.; Shen, J. L.; Tang, W. Y.; Ren, Y. J.; Wang, L. RSC Adv. 2014, 4 (107), 62262. doi: 10.1039/c4ra13353f

(39) Sun, X. F.; Xin, X.; Tang, N.; Guo, L.; Wang, L.W.; Xu, G. Y. J. Phys. Chem. B 2014, 118 (3), 824. doi: 10.1021/jp409626s

(40) Sun, X. F.; Du, Z. P.; Li, W. Z.; Xin, X.; Tang, N.; Wang, L.; Yuan, Colloids Surf. A 2014, 457, 345. doi: 10.1016/j.colsurfa.2014.06.003

(41) Piepenbrock, M. M.; Lloyd, G. O.; Clarke, N.; Steed, J. W. Chem. Rev. 2010, 110 (4), 1960. doi: 10.1021/cr9003067

(42) Fages, F. Angew. Chem. Int. Ed. 2006, 45 (11),1680. doi: 10.1002/anie.200503704

(43) Maity, M.; Maitra, U. Dalton Trans. 2017, 46 (28), 9266. doi: $10.1039 / \mathrm{c} 7 \mathrm{dt} 02177 \mathrm{a}$

(44) Ajayaghosh, A.; Chithra, P.; Varghese, R. Angew. Chem. Int. Ed. 2007, 46 (1-2), 230. doi: 10.1002/anie.200603611

(45) Clemens, Burda; Chen, X. B.; Radha, N.; Mostafa, A. E. Chem. Rev. 2005, 36 (27), 1025. doi: 10.1021/cr030063a

(46) Binnemans, K. Chem. Rev. 2009, 109 (9), 4283. doi: $10.1021 / \mathrm{cr} 8003983$

(47) Song, C. H.; Ye, Z. Q.; Wang, G. L.; Yuan, J. L.; Guan, Y. F. Chem. Eur. J. 2010, 16 (22), 6464. doi: 10.1002/chem.201000528

(48) Bünzli, J. G.; Piguet, C. Chem. Soc. Rev. 2005, 34, 1048. doi: 10.1039/B406082M

(49) Qiao, Y.; Lin, Y. Y.; Zhang, S. F.; Huang, J. B. Chem. Eur. J. 2011, 17 (18), 5180. doi: 10.1002/ chem.201003255

(50) McCoy, C. P. Chem. Mater. 2006, 18 (18), 4336. doi: $10.1021 / \mathrm{cm} 060603 \mathrm{v}$

(51) Bhowmik, S.; Banerjee, S.; Maitra, U. Chem. Commun. 2010, 46 (45), 8642. doi: $10.1039 / \mathrm{c} 0 \operatorname{cc} 02939 \mathrm{~d}$

(52) Laishram, R. Chem. Select. 2018, 3 (2), 519. doi: 10.1002/slct.201701013 
(53) Qiao, Y.; Lin, Y. Y.; Yang, Z. Y.; Chen, H. F.; Zhang, S. F.; Yan, Y.; Huang, J. B. J. Phys. Chem. B 2010,114 (36), 11725. doi: $10.1021 /$ jp 1047369

(54) Wang, Y. T.; Xin, X.; Li, W. Z.; Jia, C. Y.; Wang, L.; Shen, J. L.; Xu, G. Y. J. Colloid Interface Sci. 2014, 431, 82. doi: $10.1016 /$ j.jcis.2014.06.013

(55) Grassmann, O.; Müller, G.; Löbmann, P. Chem. Mater. 2002, 14 (11), 4530. doi: 10.1021/cm0212156

(56) Liu, J. Q.; Song, G. S.; He, C. C.; Wang, H. L. Macro. Rapid Commun. 2013, 34 (12), 1002. doi: 10.1002/marc.201300242

(57) Liu, J. H.; Chen, G. S.; Jiang, M. Macromolecules 2011, 44 (19), 7682. doi: $10.1021 / \mathrm{ma} 201620 \mathrm{w}$

(58) Zu, S. Z.; Han, B. H. J. Phys. Chem. C 2009, 113 (31), 13651. doi: $10.1021 /$ jp9035887

(59) Tanaka, Y.; Gong, J. P.; Osada, Y. Pro. Polym. Sci. 2005, 30 (1), 1. doi: 10.1016/j.progpolymsci.2004.11.003

(60) Dash, R.; Foston, M.; Ragauskas, A. Carbohydr. Polym. 2013, 91 (2), 638. doi: 10.1016/j.carbpol.2012.08.080

(61) Tong, X.; Zheng, J. L.; Lu, Y. C.; Zhang, Z. F.; Cheng, H. M. Mater. Lett. 2007, 61(8-9), 1704. doi: 10.1016/j.matlet.2006.07.115

(62) Wang, L.; Xin, X.; Yang, M. Z.; Ma, X.; Shen, J. L.; Yuan, S. L. Colloids Surf. A 2015, 483,112. doi: 10.1016/j.colsurfa.2015.07.044

(63) Raghavan, S. R.; Fritz, G.; Kaler, E. W. Langmuir 2002, 18 (10), 3797. doi: 10.1021/1a0115583

(64) Liu, S. Y.; Gonzalez, Y. I.; Kaler, E. W. Langmuir 2003, 19 (26), 10732. doi: 10.1021/la035409r

(65) Cheng, C. Y.; Oh, H.; Wang, T. Y.; Raghavan, S. R.; Tung, S. H. Langmuir 2014, 30 (34), 10221. doi: 10.1021/la502380q

(66) Cheng, C. Y.; Wang, T. Y.; Tung, S. H. Langmuir 2015, 31 (49), 13312. doi: 10.1021/ acs.langmuir.5b03267

(67) Song, Z. H.; Xin, X.; Shen, J. L.; Zhang, H.; Wang, S. B.; Yang, Y. Z. RSC Adv. 2016, 6 (4), 2966. doi: 10.1039/c5ra21979e

(68) Aida, T.; Meijer, E.; Stupp, S. Science 2012, 335 (6070), 813. doi: $10.1126 /$ science. 1205962

(69) Tao, K.; Makam, P.; Aizen, R.; Gazit, E. Science 2017, 358 (6365), 9756. doi: 10.1126/science.aam 9756

(70) Ariga, K.; Yusuke, Y.; Gaulthier, R. Chem. Lett. 2014, 45 (21), 36. doi: 10.1246/cl.130987

(71) Zhang, R. Y.; Xing, R. R.; Jiao, T. F.; Ma, K.; Chen, C. J.; Ma, G. H.; Yan, X. H. ACS Appl. Mater. Interf. 2016, 8 (21), 13262. doi: 10.1021/acsami.6b02416

(72) Xing, R. R.; Liu, K.; Jiao, T. F.; Zhang, N.; Ma, K.; Zhang, R. Y.; Zou, Q. L.; Ma, G. H.; Yan, X. H. Adv. Mater. 2016, 28 (19), 3669. doi: 10.1002/adma.201600284
(73) Ma, K.; Xing, R. R.; Jiao, T. F.; Shen, G. Z.; Chen, C. J.; Li, J. B.; Yan, X. H. ACS Appl. Mater. Inter. 2016, 8 (45), 30759. doi: 10.1021/acsami.6b10754

(74) Fichman, G.; Guterman, T.; Damron, J.; Adler-Abramovich, L.; Schmidt, J.; Kesselman, E.; Shimon, L.; Ramamoorthy, A.; Talmon, Y.; Gazit, E. Sci. Adv. 2016, 2 (2), No. e1500827. doi: $10.1126 /$ sciadv. 1500827

(75) Liyanage, W.; Brennessel, W.; Nilsson, B. Langmuir 2015, 31 (36), 9933. doi: 10.1021/acs.langmuir.5b01953

(76) Kunal, S. M.; Nicholas, P.; Feyter, D. S.; Neil, R. C. Chem. Soc. Rev. 2017, 46 (9), 2520. doi: 10.1039/b925459e

(77) Fukui, T.; Kawai, S.; Fujinuma, S.; Matsushita, Y.; Yasuda, T.; Sakurai, T.; Seki, S.; Takeuchi, M.; Sugiyasu, K. Nat. Chem. 2017, 9 (5), 493. doi: 10.1038/nchem.2684

(78) Bouju, X.; Mattioli, C.; Franc, G; Pujol, A; Gourdon, A. Chem. Rev. 2017, 117 (3), 1407. doi: 10.1021/acs.chemrev.6b00389

(79) Han, P.; Ma, N.; Ren, H. F.; Xu, H. P.; Li, Z. B.; Wang, Z. Q.; Zhang, X. Langmuir 2010, 26 (18), 14414. doi: $10.1021 / 1 \mathrm{a} 102837 \mathrm{a}$

(80) Ahmed, R.; Patra, S. K.; Hamley, I. W.; Manners, I.; Faul, C. F. J. Am. Chem. Soc. 2013, 135 (7), 2455. doi: 10.1021/ja312318d

(81) Jin, Y. D.; Bi, L. H.; Shao, Y.; Dong, S. J. Chem. Eur. J. 2004, 10 (13), 3225. doi: 10.1002/chem.200305489

(82) Li, J. F.; Chen, Z. J.; Zhou, M. C.; Jing, J. B.; Li, W.; Wang, Y.; Wu, L. X.; Wang, L. Y.; Wang, Y. Q.; Lee, M. Angew. Chem. Int. Ed. 2016, 55 (7), 2592. doi: 10.1002/anie.201511276

(83) Yan, X. H.; Zhu, P. L.; Fei, J. B.; Li, J. B. Adv. Mater. 2010, 22 (11), 1283. doi: 10.1002/adma.200901889

(84) Kumar, S.; Singh, P.; Mahajan, A.; Kumar, S. Org. Lett. 2013, 15 (13), 3400. doi: 10.1021/ol401452t

(85) MacLachlan, M. J.; Coombs, N.; Ozin, G. A. Nature 1999, 397 (6721), 681. doi: 10.1038/17776

(86) Zhang, H.; Guo, L.Y.; Jiao, J. M.; Xin, X.; Sun, D.; Yuan, S. L. ACS Sustainable Chem. Eng. 2017, 5 (2), 1358. doi: 10.1021/acssuschemeng.6b01805

(87) Xia, C. X.; Wang, Z.; Sun, D.; Jiang, B. L.; Xin, X. Langmuir 2017, 33 (46), 13242. doi: 10.1021/acs.langmuir.7b03495

(88) Zhu, L. J.; Huang, D. D.; Li, Q. T.; Xu, G. T.; Chen. X. Acta Phys. -Chim. Sin. 2013, 29 (11), 2415. [朱丽杰, 黄丹丹, 李钦堂, 许冠辰, 陈晓. 物理化学学报, 2013, 29 (11), 2415] doi: 10.3866/PKU.WHXB201309243

(89) Qiao, Y.; Lin, Y. Y.; Wang, Y. J.; Yang, Z. Y.; Liu, J.; Zhou, J.; Yan, Y.; Huang, J. B. Nano Lett. 2009, 9 (12), 4500. doi: $10.1021 / \mathrm{nl} 9028335$

(90) Jiao, J. M.; Xin, X.; Wang, X. G.; Xie, Z. C.; Xia, C. X.; Pan, W. $R S C A d v$. 2017, 7 (69), 43474. doi: 10.1039/c7ra06592b 
(91) Li, G. H.; Wang, Y. T.; Wang, L.; Song, A. X.; Hao, J. C. Langmuir 2016, 32 (46), 12100. doi: 10.1021/acs.langmuir.6b03052

(92) Wang, Q. L.; Li, W. N.; Shi, J. S. RSC Adv. 2015, 5 (75), 61330. doi: $10.1039 / \mathrm{c} 5 \mathrm{ra} 07580 \mathrm{~g}$

(93) Song, Z. H.; Xin, X.; Shen, J. L.; Zhang, H.; Wang, S. B.; Yang, Y. Z. J. Mater. Chem. C 2016, 4 (36), 8439. doi: $10.1039 / \mathrm{C} 6 \mathrm{TC} 02329 \mathrm{~K}$
(94) Gao, Y. Y.; Zhang, Q. Y.; Wang, L.; Feng, L.; Gao, L.; Zhang, G. P.; Xia, C. X.; Xin, X. Colloids Surf. A 2018, 546, 276. doi: 10.10 16/j.colsurfa.2018.03.009

(95) Jiao, J. M.; Shen, J. L.; Wang, L.; Xie, Z. C.; Xia, C. X.; Xin, X. Colloids Surf. A 2018, 45, 8. doi: 10.1016/j.colsurfa.2018.02.013

(96) Song, Z. H.; Xin, X.; Xia, C. X.; Sun, P. P.; Cheng, X. H.; Xiang, Z. Y.; Yang, Y. Z. J. Mol. Liq. 2018, 255, 1. doi: 10.1016/j.molliq.2018.01.13 